\title{
DYNAMIC CHARACTERISTICS OF CYLINDRICAL SHELLS CONSIDERING FLUID-STRUCTURE INTERACTION
}

\author{
MYUNG JO JHUNG ${ }^{*}$, WAL TAE KIM and YONG HO RYU \\ Safety Research Division, Korea Institute of Nuclear Safety \\ 19 Guseong-dong, Yuseong-gu, Daejeon, 305-338, Korea \\ 'Corresponding author. E-mail : mjj@kins.re.kr
}

Received August 3, 2009

Accepted for Publication September 16, 2009

To assure the reliability of cylinders or shells with fluid-filled annulus, it is necessary to investigate the modal characteristics considering fluid-structure interaction effect. In this study, theoretical background and several finite element models are developed for cylindrical shells with fluid-filled annulus considering fluid-structure interaction. The effect of the inclusion of the fluid-filled annulus on the natural frequencies is investigated, which frequencies are used for typical dynamic analyses such as responses spectrum, power spectral density and unit load excitation. Their response characteristics are addressed with respect to the various representations of the fluid-structure interaction effect.

KEYWORDS : Cylindrical Shell, Fluid-structure Interaction, Annulus, Modal Characteristic, Response Spectrum, Power Spectral Density, Harmonic Analysis, Added Mass

\section{INTRODUCTION}

Coaxial shells or cylinders containing fluid have been widely used as structural components in various applications. Examples are reactor internal structures such as core support barrel and upper guide structure barrel coupled with each other by fluid-filled annulus [1] and spent fuel storage racks. To assure the reliability of those components during normal operations of a nuclear power plant, it is necessary to predict vibration amplitude, necessitating the investigation of the modal characteristics considering fluid-structure interaction effect.

There are many finite element models used for assessing the structural integrity of cylinders or shells with fluidfilled annulus. In the past, the fluid region was not modeled explicitly and its mass was added to the structural mass in the form of an added mass for simplicity. In this case the fluid-structure interaction effect, the so called annulus effect, is not considered in the analysis, generating unrealistic or unconservative results in some cases. Therefore, it is necessary to make a 3-dimensional model including the fluid region and to couple the two nodes that are assigned to the fluid and the structure. Fortunately, commercial programs such as ANSYS [2] can easily model couplings between the fluid and the structure and can consider the annulus effect for various types of analyses very efficiently.

Therefore, in this study, theoretical background and several finite element models are developed for coaxial cylindrical shells with fluid-filled annulus considering fluid-structure interaction. The effect of the inclusion of the fluid-filled annulus on the natural frequencies is investigated by comparing frequencies between various finite element models. Using the modal characteristics, typical dynamic analyses such as responses spectrum, power spectral density (PSD) and unit load excitation analysis are performed and their response characteristics are addressed with respect to the various representations of the fluid-structure interaction.

\section{THEORETICAL DEVELOPMENT}

The equations of motion can be represented for the displacements $x$ on the structure as:

$$
\begin{aligned}
& {\left[\begin{array}{ll}
m_{a c a} & m_{a b} \\
m_{b a} & m_{b b}
\end{array}\right]\left[\begin{array}{l}
\ddot{x}_{a} \\
\ddot{x}_{b}
\end{array}\right]+\left[\begin{array}{ll}
c_{a a b} & c_{a b} \\
c_{b a} & c_{b b}
\end{array}\right]\left[\begin{array}{c}
\dot{x}_{a} \\
\dot{x}_{b}
\end{array}\right]} \\
& +\left[\begin{array}{ll}
k_{a b a} & k_{a b} \\
k_{b b i} & k_{b b}
\end{array}\right]\left[\begin{array}{l}
x_{a} \\
x_{b}
\end{array}\right]=\left[\begin{array}{c}
0 \\
p_{b}
\end{array}\right]
\end{aligned}
$$

where the displacements $x_{b}$ are forced to vary in a defined manner with prescribed functions of time and $p_{b}$ represents the column matrix of unknown forces causing the displacements $x_{b}$.

If the hydrodynamic couplings are considered in the analysis, the mass matrix is 


$$
M=M_{s}+M_{h}=\left[\begin{array}{cc}
m_{a a s} & 0 \\
0 & m_{b b s}
\end{array}\right]+\left[\begin{array}{cc}
m_{a a h} & m_{a b} \\
m_{b u} & m_{b h h}
\end{array}\right]
$$

where $M_{s}$ and $M_{h}$ are the structural and hydrodynamic mass matrices, respectively. Considering two mass points with hydrodynamic couplings, Equation (1) becomes

$$
\left[\begin{array}{cc}
m_{a a s}+m_{a a h t} & m_{a b} \\
m_{b a} & m_{b b s}+m_{b b h}
\end{array}\right]\left[\begin{array}{c}
\ddot{x}_{a} \\
\ddot{x}_{b}
\end{array}\right]=\left[\begin{array}{c}
F_{a} \\
F_{b}
\end{array}\right]
$$

where $F_{a}$ and $F_{b}$ are the spring and damping forces at nodes $a$ and $b$ defined as:

$$
\left[\begin{array}{l}
F_{a} \\
F_{b}
\end{array}\right]=\left[\begin{array}{c}
-\left(c_{a a} \dot{x}_{a}+c_{a b} \dot{x}_{b}+k_{a a} x_{a}+k_{a b} x_{b}\right) \\
-\left(c_{b a} \dot{x}_{a}+c_{b b} \dot{x}_{b}+k_{b a} x_{a}+k_{b b} x_{b}\right)+p_{b}
\end{array}\right]
$$

In a solution which is based on a direct integration of the equations of motion, the spring and damping forces are evaluated at each instant of time and then the accelerations are solved. Integration of the accelerations gives the velocities and displacements needed to reevaluate the accelerations for the next time step [3].

The hydrodynamic mass matrix can be calculated from the fluid velocity potential for the two long concentric cylinders separated by a gap filled with ideal and compressible fluid, as shown in Figure 1. The governing continuity equation is written for any instant [4] as:

$$
\frac{\partial^{2} \phi}{\partial r^{2}}+\frac{1}{r} \frac{\partial \phi}{\partial r}+\frac{1}{r^{2}} \frac{\partial^{2} \phi}{\partial \theta^{2}}=0
$$

where $\phi$ is the velocity potential, and $r$ and $\theta$ are the radial and angular coordinates. Applying the boundary conditions for the radial component of fluid velocity at $r=a$ and $r=$ $b$ yields the following solution for $\phi$ :

$$
\phi=\frac{b^{2} a^{2}}{b^{2}-a^{2}}\left\{\left(\frac{\dot{x}_{j}}{a^{2}}-\frac{\dot{x}_{i}}{b^{2}}\right) r+\frac{\dot{x}_{j}-\dot{x}_{i}}{r}\right\} \cos \theta
$$

Therefore, fluid forces on the cylinders are obtained by integrating fluid pressure in the annulus along the circumference, resulting in matrix form as:

$$
M_{h}=\left[\begin{array}{cc}
m_{1} f_{c} & -m_{1}\left(f_{c}+1\right) \\
-m_{1}\left(f_{c}+1\right) & m_{2} f_{c}
\end{array}\right]
$$

where $m_{1}\left(=\rho \pi a^{2}\right)$ is the mass of fluid displaced by the inner cylinder, $m_{2}\left(=\rho \pi b^{2}\right)$ is the mass of fluid contained by the outer cylinder and $f_{c}$ is the magnification factor for hydrodynamic mass depending on the size of the annulus defined as:

$$
f_{c}=\frac{b^{2}+a^{2}}{b^{2}-a^{2}}
$$

If the gap is infinite, the magnification factor $f_{c}$ becomes zero and only the off-diagonal terms exist with the added mass of the inner cylinder, as shown in Figure 2. If the

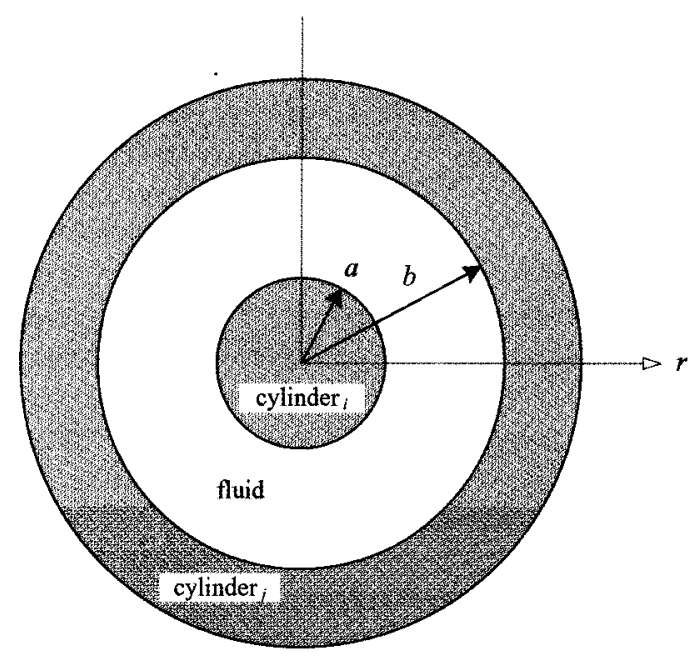

Fig. 1. Coaxial Cylinders with Fluid Coupling

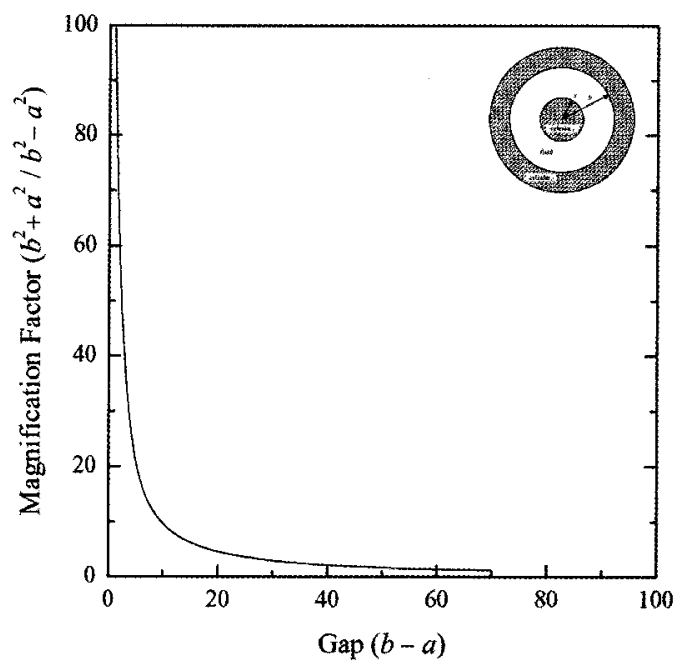

Fig. 2. Magnification Factor of Hydrodynamic Mass for Two Coaxial Cylinders 
gap is so small, the factor becomes infinite and the fluid force tends to cushion one cylinder from the other [5].

\subsection{Damping Values}

A viscous damping matrix $C$ formed by a linear combination of the mass and stiffness matrices is used [6]:

$$
C=\alpha M+\beta K
$$

where $M$ is the mass matrix and $K$ the stiffness matrix. In this form, $\alpha$ and $\beta$ are constants to be determined from the two given damping ratios that correspond to two unequal frequencies of vibration. Much of the experimental data on damping properties consists of ratios, $\xi_{i}$, of natural damping to critical damping for a particular mode of vibration, $i$. If a natural frequency $\omega_{i}$ and a modal damping ratio $\xi_{i}$ are selected, the mass and stiffness matrix multipliers for damping, $\alpha$ and $\beta$, should satisfy the following relation [7]:

$$
\xi_{i}=\frac{\alpha}{2 \omega_{i}}+\frac{\beta \omega_{i}}{2}
$$

For a given damping ratio $\left(\xi_{i}\right.$ and $\left.\xi_{j}\right)$ and a frequency range $\left(\omega_{i}\right.$ and $\left.\omega_{j}\right)$, two equations can be solved simultaneously for the values of $\alpha$ and $\beta$, as follows:

$$
\begin{gathered}
\alpha=\frac{2 \omega_{i} \omega_{j}\left(\omega_{i} \xi_{j}-\omega_{j} \xi_{i}\right)}{\omega_{i}^{2}-\omega_{j}^{2}} \\
\beta=\frac{2\left(\omega_{i} \xi_{i}-\omega_{j} \xi_{j}\right)}{\omega_{i}^{2}-\omega_{j}^{2}}
\end{gathered}
$$

Then for an arbitrary frequency $\omega_{r}$, the damping ratio $\xi_{r}$ can be computed by eliminating $\alpha$ and $\beta$ and is given as:

$$
\xi_{r}=\frac{1}{\omega_{i}^{2}-\omega_{j}^{2}}\left[\begin{array}{l}
\frac{\omega_{i} \omega_{j}\left(\omega_{i} \xi_{j}-\omega_{j} \xi_{i}\right)}{\omega_{r}} \\
+\omega_{r}\left(\omega_{i} \xi_{i}-\omega_{j} \xi_{j}\right)
\end{array}\right]
$$

where the first and second terms in Equation (13) represent mass damping and stiffness damping, respectively. Mass damping introduces the damping forces that are proportional to the velocities of each mass point in the system and may be used to represent the energy loss due to impact and friction. Stiffness damping introduces the damping forces that are proportional to the time rate of deformation. While the mass damping introduces the damping forces due to the rigid body motion of the system, the stiffness damping does not.

\section{FINITE ELEMENT MODELS}

Consider fluid-filled coaxial cylindrical shells with clamped-free boundary conditions at bottom and top ends. The cylindrical shells have mean radii $R_{1}$, and $R_{2}$, height $L$, and wall thickness $h$. The inner and outer shells are coupled with a fluid-filled annular gap. The inner cylindrical shell has a mean radius of $100 \mathrm{~mm}$, a length of $300 \mathrm{~mm}$, and a wall thickness of $2 \mathrm{~mm}$. The outer cylindrical shell has a mean radius of $150 \mathrm{~mm}$ with the same length and wall thickness. The physical properties of the shell material are as follows: Young's modulus $=69.0 \mathrm{GPa}$, Poisson's ratio $=0.3$, and mass density $=2700 \mathrm{~kg} / \mathrm{m}^{3}$. Water is used as the contained fluid with a density of $1000 \mathrm{~kg} / \mathrm{m}^{3}$. The

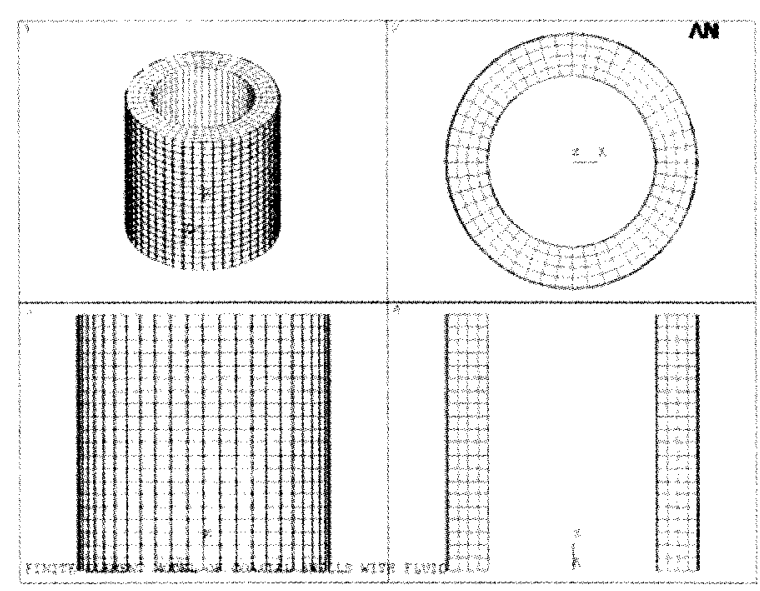

(b) 3-D model

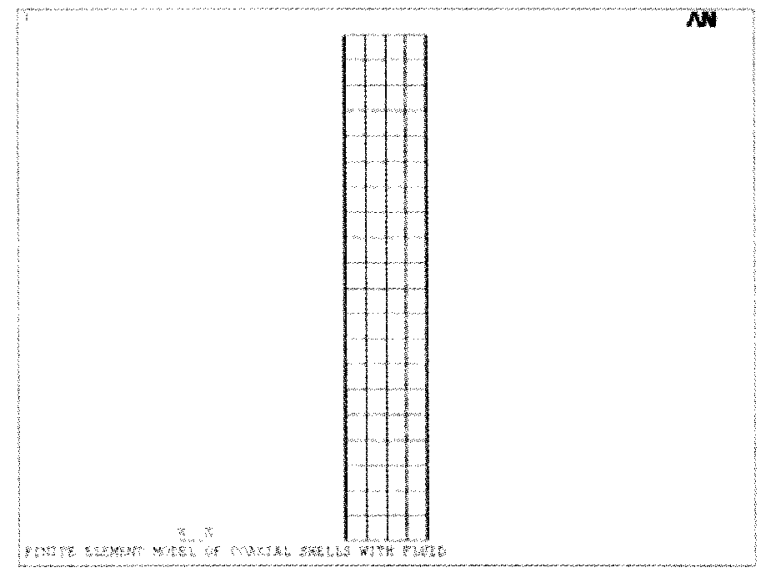

(b) 2-D model

Fig. 3. Finite Element Models of Coaxial Cylindrical Shells with Fluid 
sound speed in water, $1483 \mathrm{~m} / \mathrm{s}$, is equivalent to the bulk modulus of elasticity, $2.2 \mathrm{GPa}$

A three-dimensional model is constructed for the finite element analysis. The fluid region is divided into a number of identical 3-dimensional contained fluid elements (FLUID80) with eight nodes, having three degrees of freedom at each node. The fluid element FLUID80 is particularly well suited for calculating hydrostatic pressures and fluid/solid interactions. The circular cylindrical shell is modeled as elastic shell elements (SHELL63) with four nodes. The model has 3840 (radially $4 \times$ axially $20 \times$ circumferentially 48) fluid elements and 1920 shell elements, as shown in Figure 3(a).

Another finite element model is constructed using axisymmetric-harmonic structural shell elements (SHELL61) and axisymmetric-harmonic contained fluid elements (FLUID81) for cylinder and fluid, respectively. The model has 80 fluid elements and 40 shell elements, as shown in Figure 3(b).

The nodes connected entirely by the fluid elements are free to move arbitrarily in three-dimensional space, with the exception of those that are restricted to motion in the bottom surface of the fluid cavity. The radial velocities of the fluid nodes along the wetted shell surfaces coincide with the corresponding velocities of the shells. For the shell, clamped-free boundary condition is considered at bottom and top ends.

If the fluid is not included in the model, the mass matrix in Equation (2) is

$$
M=\left[\begin{array}{cc}
m_{a a s} & 0 \\
0 & m_{b t s}
\end{array}\right]
$$

If the fluid is included as an added mass only in the model, the mass matrix in Equation (2) is

$$
M=\left[\begin{array}{cc}
m_{a a s}+m_{1} f_{c} & 0 \\
0 & m_{b h s}+m_{2} f_{c}
\end{array}\right]
$$

If the fluid is included fully as hydrodynamic coupling in the model, the mass matrix in Equation (2) is

$$
M=\left[\begin{array}{cc}
m_{a a s}+m_{1} f_{c} & -m_{1}\left(f_{c}+1\right) \\
-m_{1}\left(f_{c}+1\right) & m_{b b s}+m_{2} f_{c}
\end{array}\right]
$$

These three different representations of the mass matrix may be the major reasons for the different modal characteristics, generating corresponding response differences due to response spectrum, harmonic, power spectral density and transient analyses.

\section{ANALYSIS}

Finite element analyses using the commercial computer code ANSYS 11.0 [2] are performed to find the natural frequencies of the coaxial circular cylindrical shells with a bounded compressible fluid.

Several cases of the finite element analyses are performed depending on the model and fluid existence. The Block Lanczos method is used for the eigenvalue and eigenvector extractions to calculate sufficient number of frequencies including fluid modes [8]. It uses the Lanczos algorithm where the Lanczos recursion is performed with a block of vectors. This method is as accurate as the subspace method, but faster. The Block Lanczos method is especially powerful when searching for eigenfrequencies in a given part of the eigenvalue spectrum of a given system. The convergence rate of the eigenfrequencies will be about the same when extracting modes in the midrange and higher end of the spectrum as when extracting the lowest modes.

Response spectrum analysis is performed for the arbitrary spectra. Single point response spectrum is applied to the bottom nodes of the shells, which are fixed in all degrees of freedom. The responses such as maximum deflections and equivalent stresses are investigated for various models.

Power spectral density analysis is performed for the arbitrary PSD. Single point PSD is applied to the bottom of the shells. The velocity PSDs of top nodes for inner and outer shells are generated for various models.

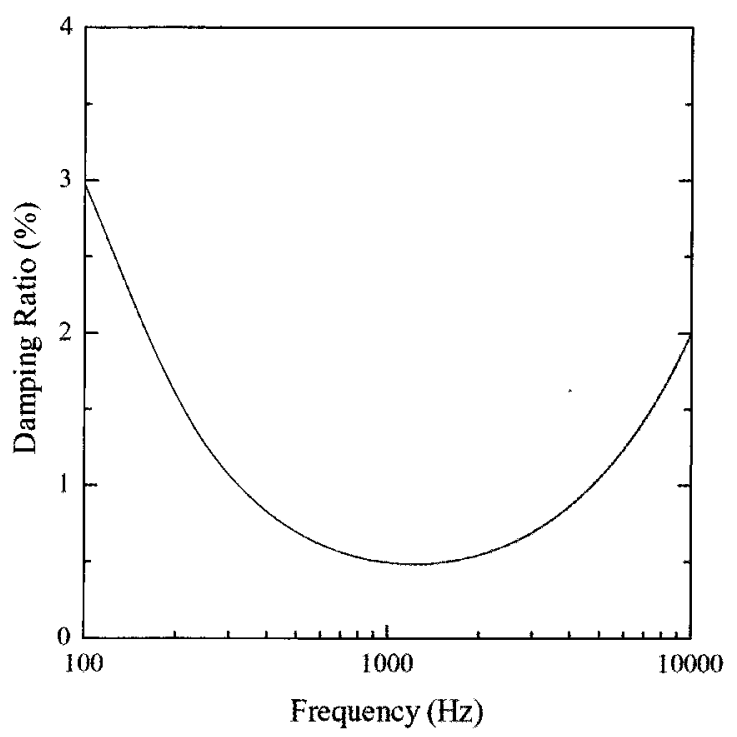

Fig. 4. Damping Ratio Used in the Transient Analysis 
A unit pulse is applied to the arbitrary node of the outer shell for the 3-dimensional model and the 2-dimensional model. The response of displacement is generated for the node where the pulse is applied. The viscous damping is assumed and is calculated. The frequency range is designated from $150 \mathrm{~Hz}$ to $10000 \mathrm{~Hz}$ to encompass the whole frequency range of the models. For the damping ratio of $2 \%, \alpha$ and $\beta$, the mass and stiffness matrix multipliers for damping, are calculated as 5.9113 and 3.9409 E- 6 by Equations (11) and (12), respectively. For an arbitrary frequency, the damping ratio can be computed as shown in Figure 4.

A unit load is applied to the arbitrary node of the outer shell. The responses of displacement and velocity are generated for the node where the unit load is applied.

\section{RESULTS AND DISCUSSION}

Mode shapes of the fluid-coupled coaxial shells are obtained by the finite element method; a typical mode is plotted in Figure 5, which shows the deformed mode shape of the fluid and shell elements for the circumferential wave number $n=3$. The dotted lines in the figures represent the undeformed shapes of the cylindrical shells.

All of the mode shapes can be classified into two mode categories according to the relative moving directions between the inner and outer shells during the vibration: in-phase mode (Figure 6) and out-of-phase mode (Figure 7). Reviewing the vibrational mode shapes revealed that as the circumferential mode number increases, the out-ofphase and in-phase modes in the serial vibrational modes appear alternatively. Mode shapes of 2-dimensional axisymmetric model are shown in Figure 8 for circumferential mode $n=1$.

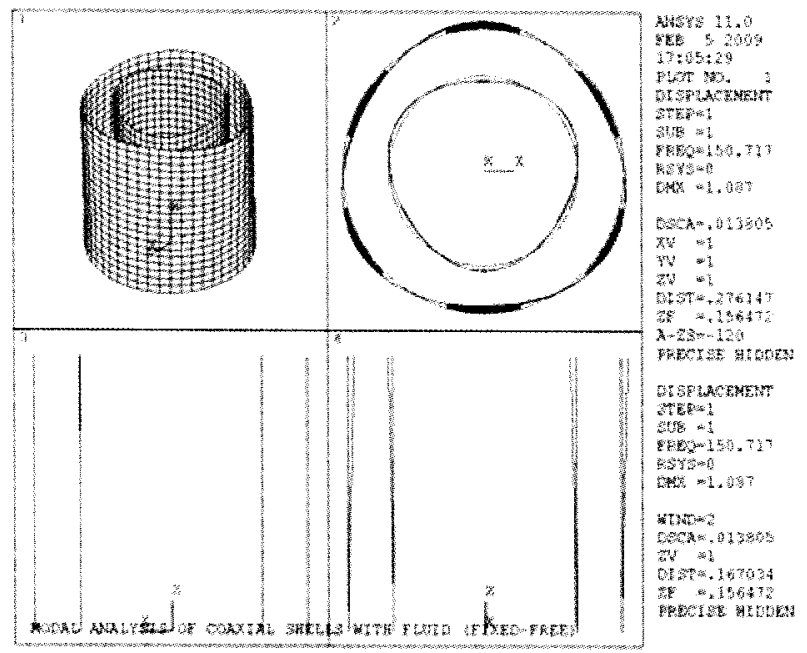

Fig. 5. Typical Mode Shape of Fluid-coupled Coaxial Shells
The frequencies between coaxial shells with and without fluid-filled annulus are compared as shown in Figures 9 and 10 . In the case of shells without fluid-filled annulus, the inner and outer shells give similar frequencies for lower axial and circumferential mode numbers, but as the circumferential mode number increases the frequencies of the inner shell are found to be larger than those of outer shell frequencies. Contrary to this, for the case of shells with fluid-filled annulus, the frequencies of the in-phase mode are always higher than those of the out-of phase mode as the frequency deviates from its lowest point.

The effect of fluid-filled annulus on the frequencies can be assessed using the normalized frequency, defined

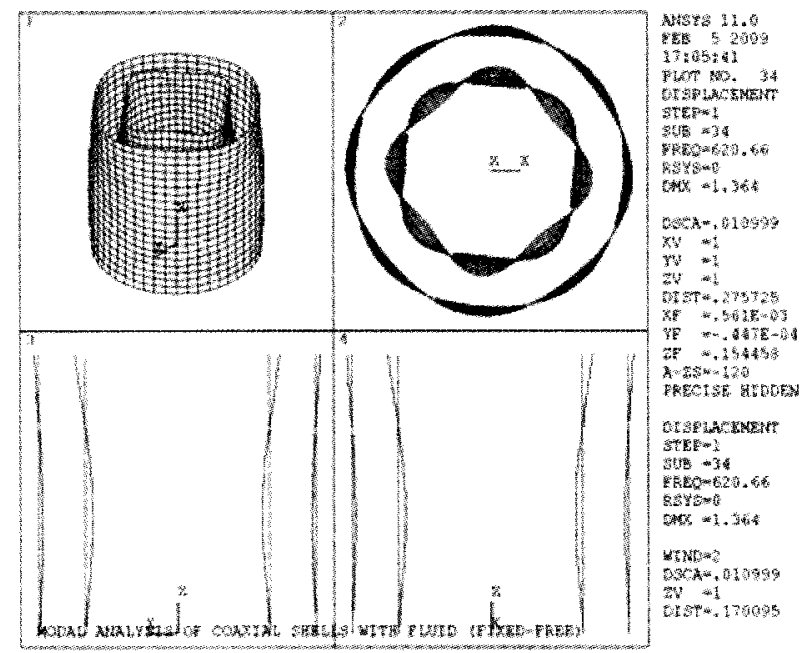

Fig. 6. Typical Mode Shape of In-phase Mode

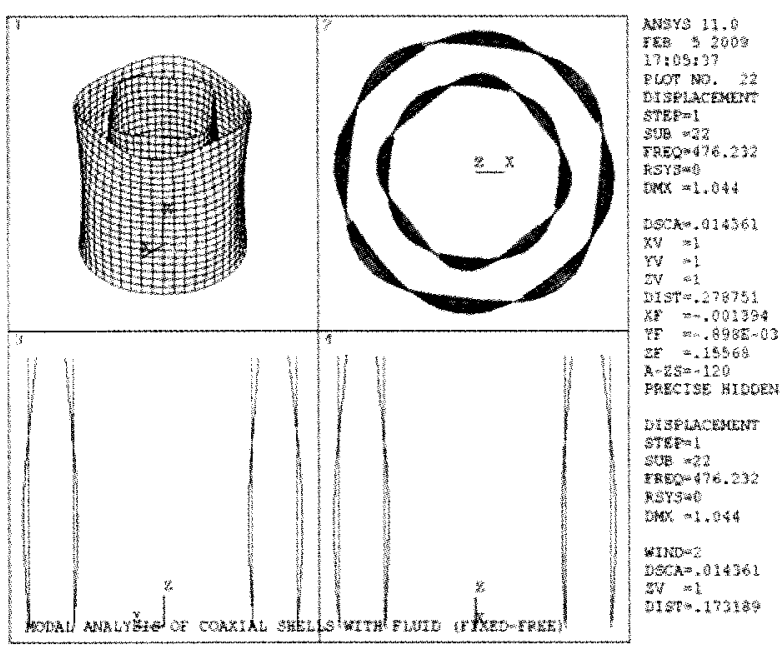

Fig. 7. Typical Mode Shape of Out-of-phase Mode 

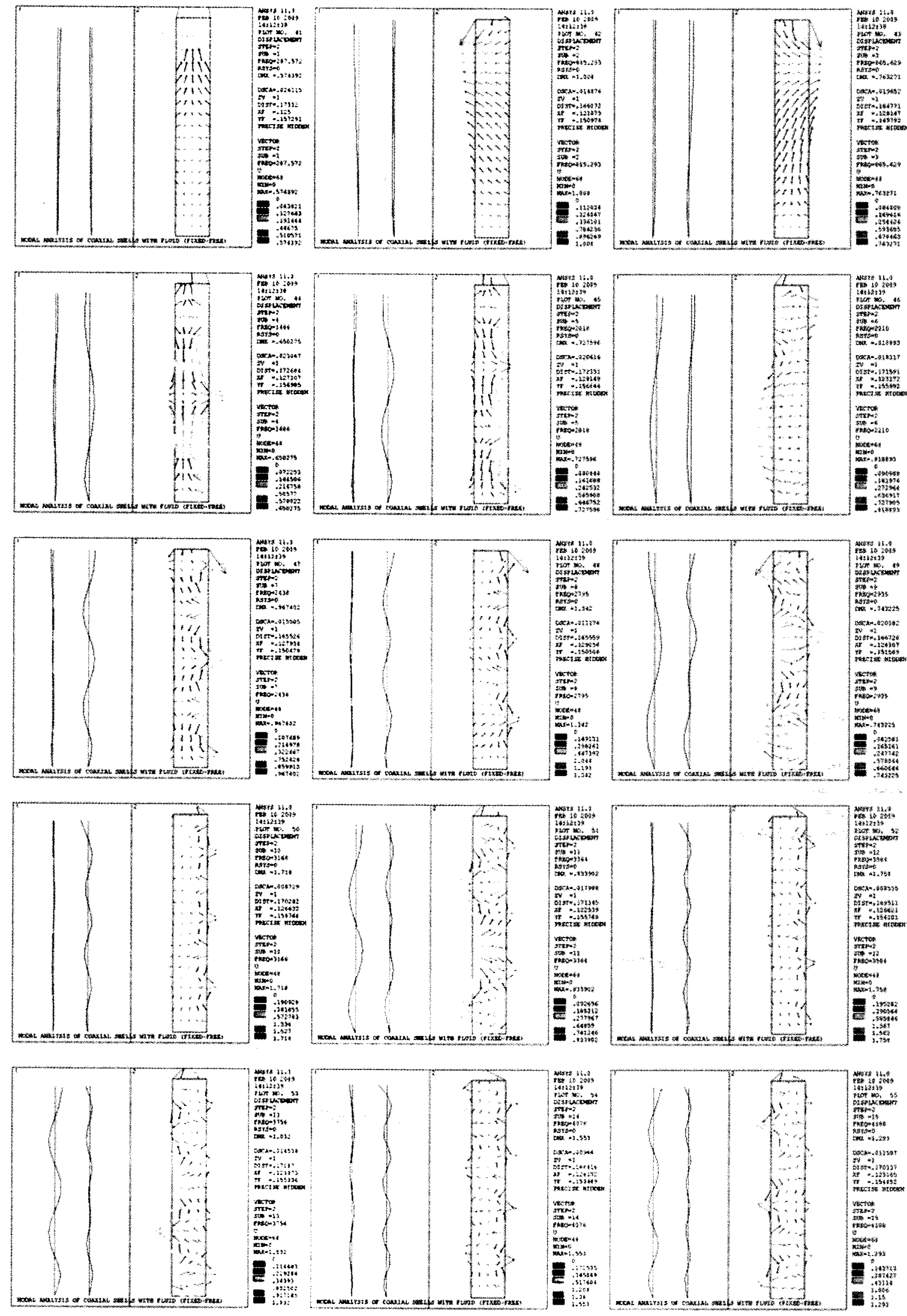

Fig. 8. Typical Mode Shapes by 2-D Model 

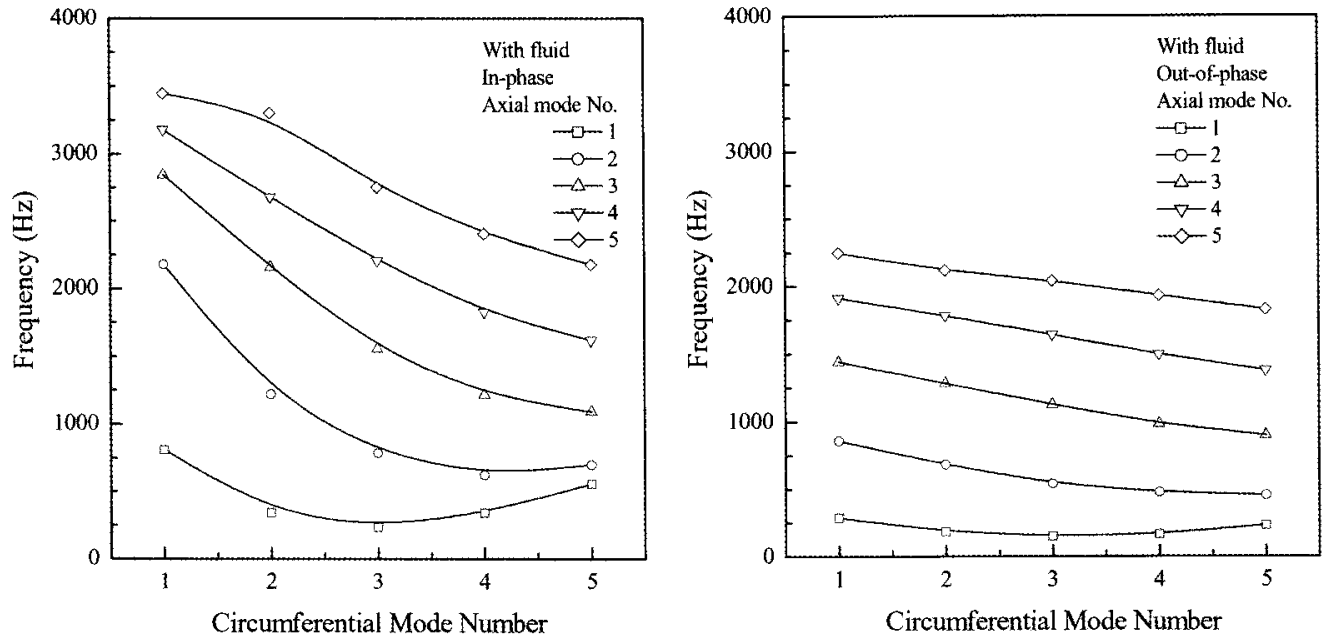

Fig. 9. Frequencies of Shells with Fluid
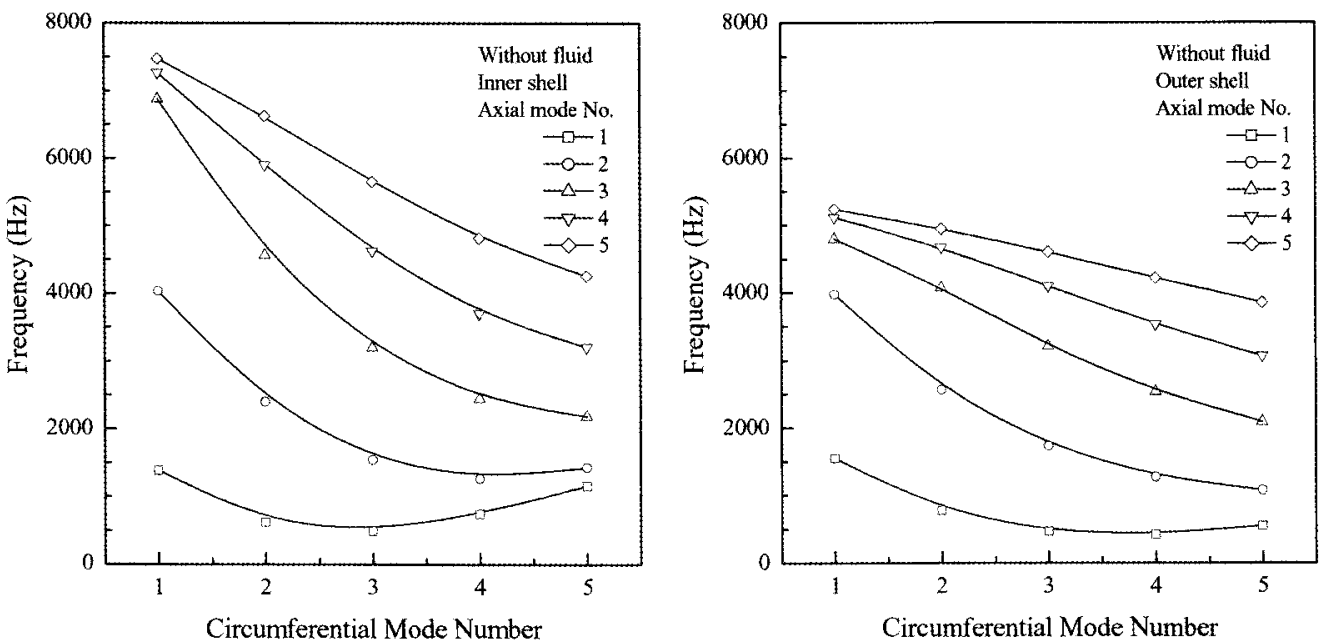

Fig. 10. Frequencies of Shells without Fluid
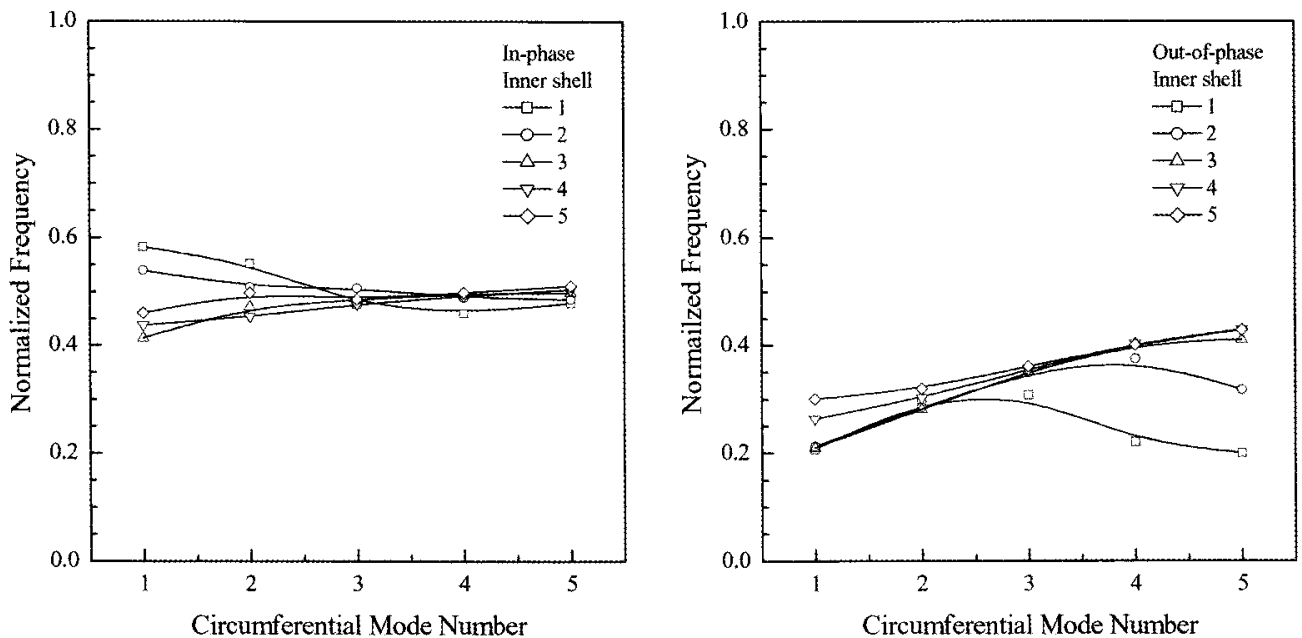

Fig. 11. Normalized Frequencies of Shells with Fluid w.r.t. without Fluid (Inner Shell) 

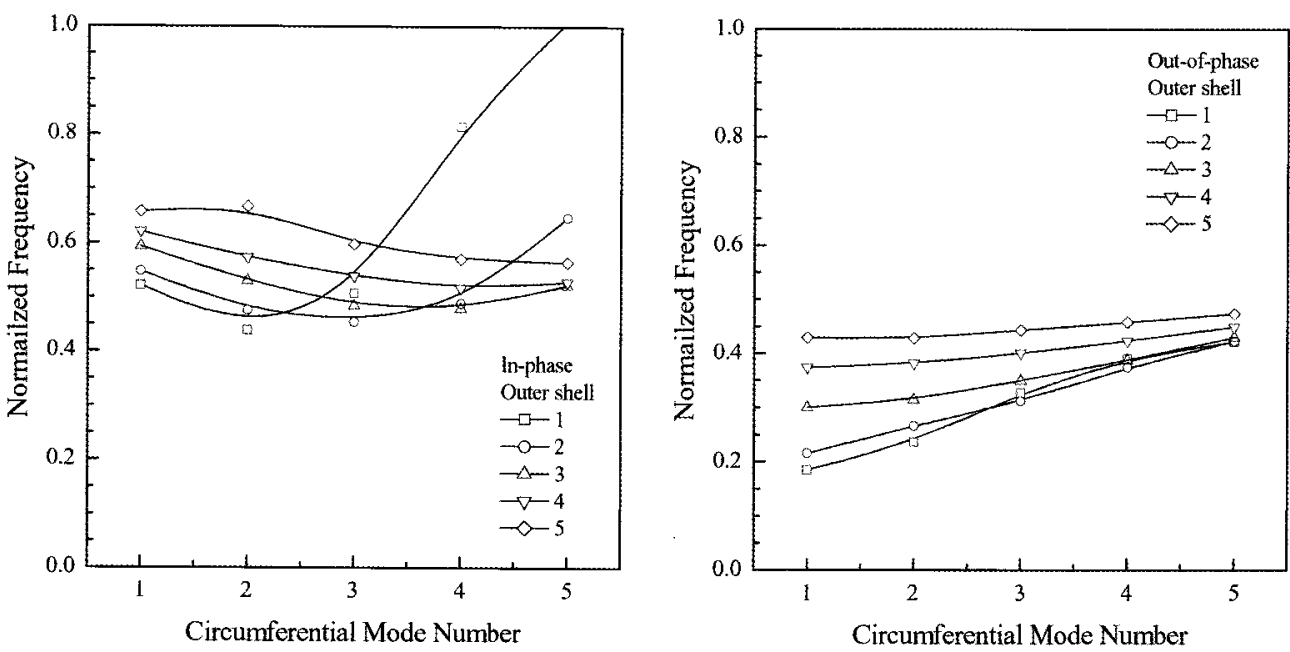

Fig. 12. Normalized Frequencies of Shells with Fluid w.r.t. without Fluid (Outer Shell)
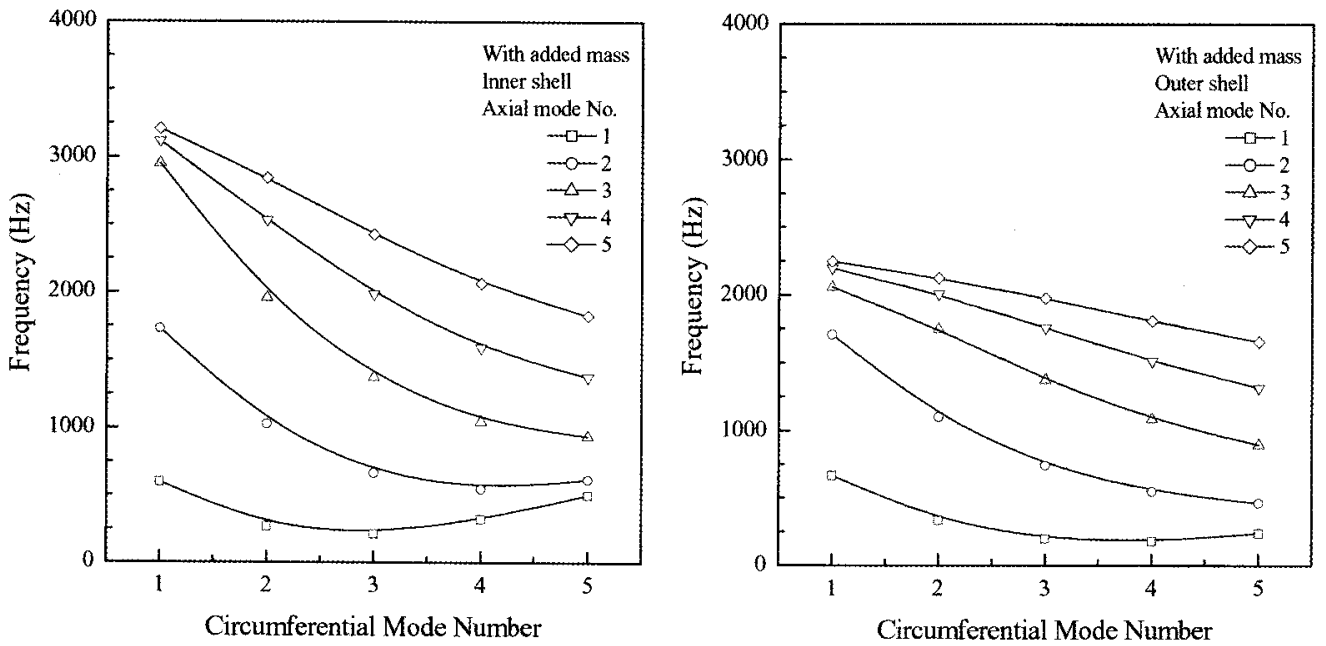

Fig. 13. Frequencies of Shells with Added Mass for Fluid

as the frequency with fluid-filled annulus divided by the frequency without it. Figures 11 and 12 show the normalized natural frequencies of fluid-filled shells for in-phase and out-of-phase modes with respect to the inner shell and outer shell in air conditions, respectively. This is based on the fact that generally in-phase modes have more deflections of inner shell and out-of-phase modes have more deflections of outer shell. The frequencies of the outer shell decrease more than those of inner shell; the reduction rate ranges from 0.4 to 0.6 for the inner shell and from 0.2 to 0.5 for the outer shell. Also, the lower circumferential modes are more affected by the inclusion of the fluid-filled annulus for the outer shell, but the inner shell has almost the same reduction rate all through the circumferential modes.

If the fluid is considered as an added mass only, the frequencies are as shown in Figure 13. In comparing frequencies between full consideration and added mass model, several modes did not appear in the added mass model, which indicates that several modes are missing due to the nonexistence of the coupling effect when the fluid is considered as an added mass only. This may lead to the different response characteristics in the ensuing dynamic analysis, such as response spectrum, PSD and harmonic analyses. The normalized frequencies in this case have the same trend as that in the full modeling case, with several exceptions as shown in Figure 14.

The frequencies and their normalized values of the 2dimensional axisymmetric model are also shown in Figures 13 and 14. The comparisons between 3-dimensional and 2 -dimensional models are shown in Figure 15. There is good agreement between them for the first several axial modes, indicating that the axisymmetric-harmonic element gives almost the same modal characteristics as the 3 dimensional element. 

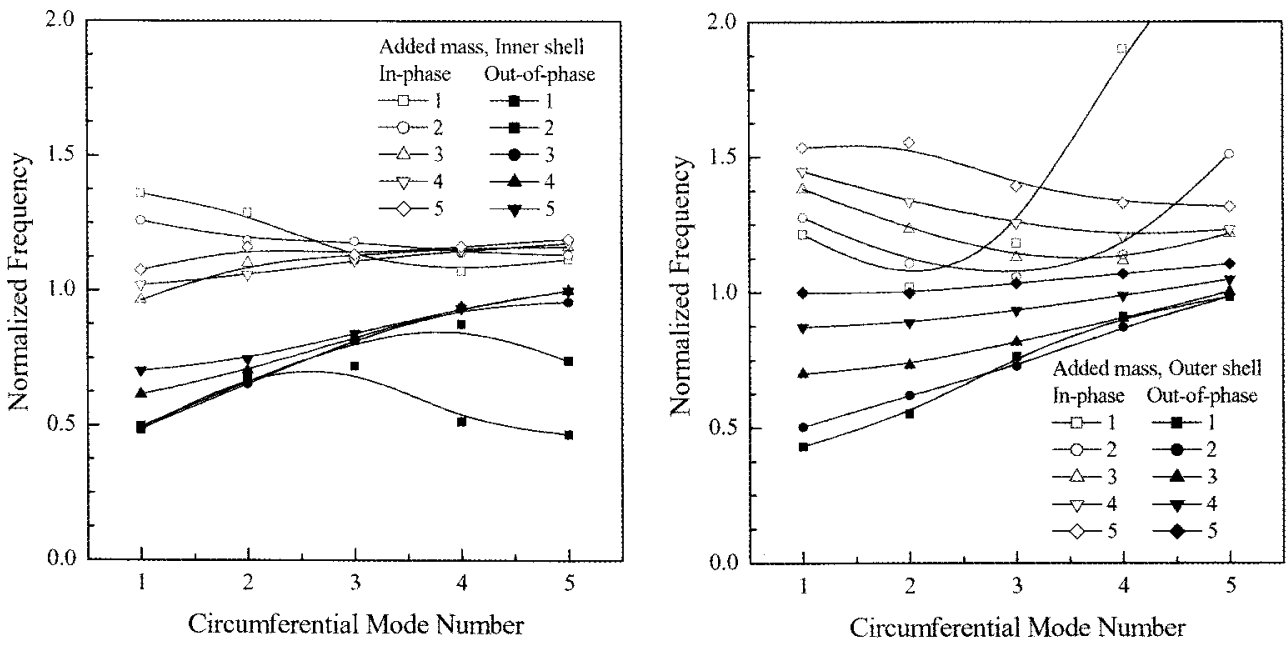

Fig. 14. Normalized Frequencies of Shells with Fluid w.r.t. without Fluid (Added Mass)
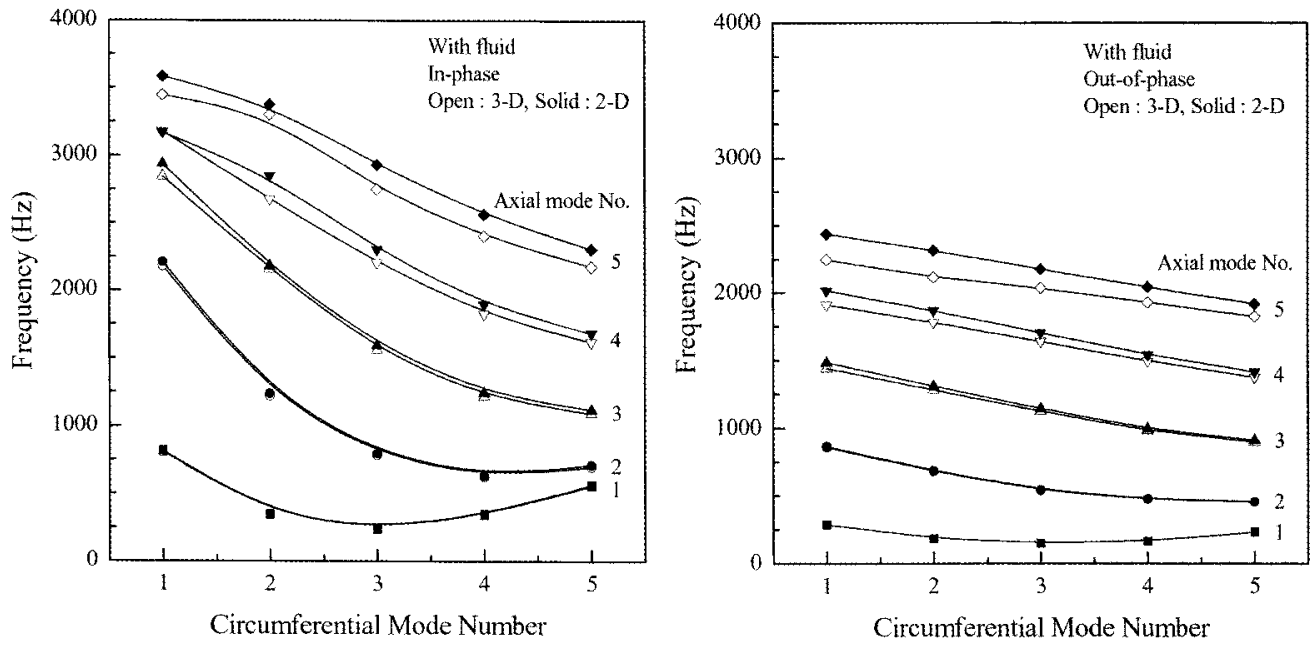

Fig. 15. Comparison of Frequencies between 3-D and 2-D Models

The maximum deflections and equivalent stresses derived from the response spectrum analysis are shown in Table 1. As indicated in the table, the responses decrease significantly when the fluid is not included in the model. If the fluid is considered as added mass only, or if the fluid coupling effect is not considered in the model, the responses are generally lowered. Therefore, it is concluded that failing to consider fluid coupling may give unconservative results, which should not be missed in the structural integrity assessment for the nuclear components.

The velocity PSDs at nodes for inner and outer shells are shown in Figure 16, which shows that there are wide differences of response PSDs between models. The amplitude and frequency depend on the modal characteristics, which also depend on the existence of fluid and the modeling technique. The maximum deflections and equivalent stresses from the PSD analysis are shown in Table 2. As
Table 1. Response Summaries from Response Spectrum Analysis

\begin{tabular}{l|c|c|c|c}
\hline \multirow{2}{*}{ Model } & \multicolumn{2}{|c|}{ Maximum deflection (m) } & \multicolumn{2}{c}{ Maximum equivalent stress (Pa) } \\
\cline { 2 - 5 } & Inner shell & Outer shell & Inner shell & Outer shell \\
\hline With fluid & $.256 \mathrm{E}-4$ & $.170 \mathrm{E}-4$ & $.495 \mathrm{E} 7$ & $.453 \mathrm{E} 7$ \\
\hline Without fluid & $.184 \mathrm{E}-5$ & $.114 \mathrm{E}-5$ & $.304 \mathrm{E} 6$ & $.185 \mathrm{E} 6$ \\
\hline $\begin{array}{l}\text { With added } \\
\text { mass for fluid }\end{array}$ & $.217 \mathrm{E}-4$ & $.171 \mathrm{E}-4$ & $.373 \mathrm{E} 7$ & $.284 \mathrm{E} 7$ \\
\hline
\end{tabular}

indicated in the table, the deflection and stress increase significantly when the fluid is included as an added mass in the model. If the fluid is not considered in the model, 

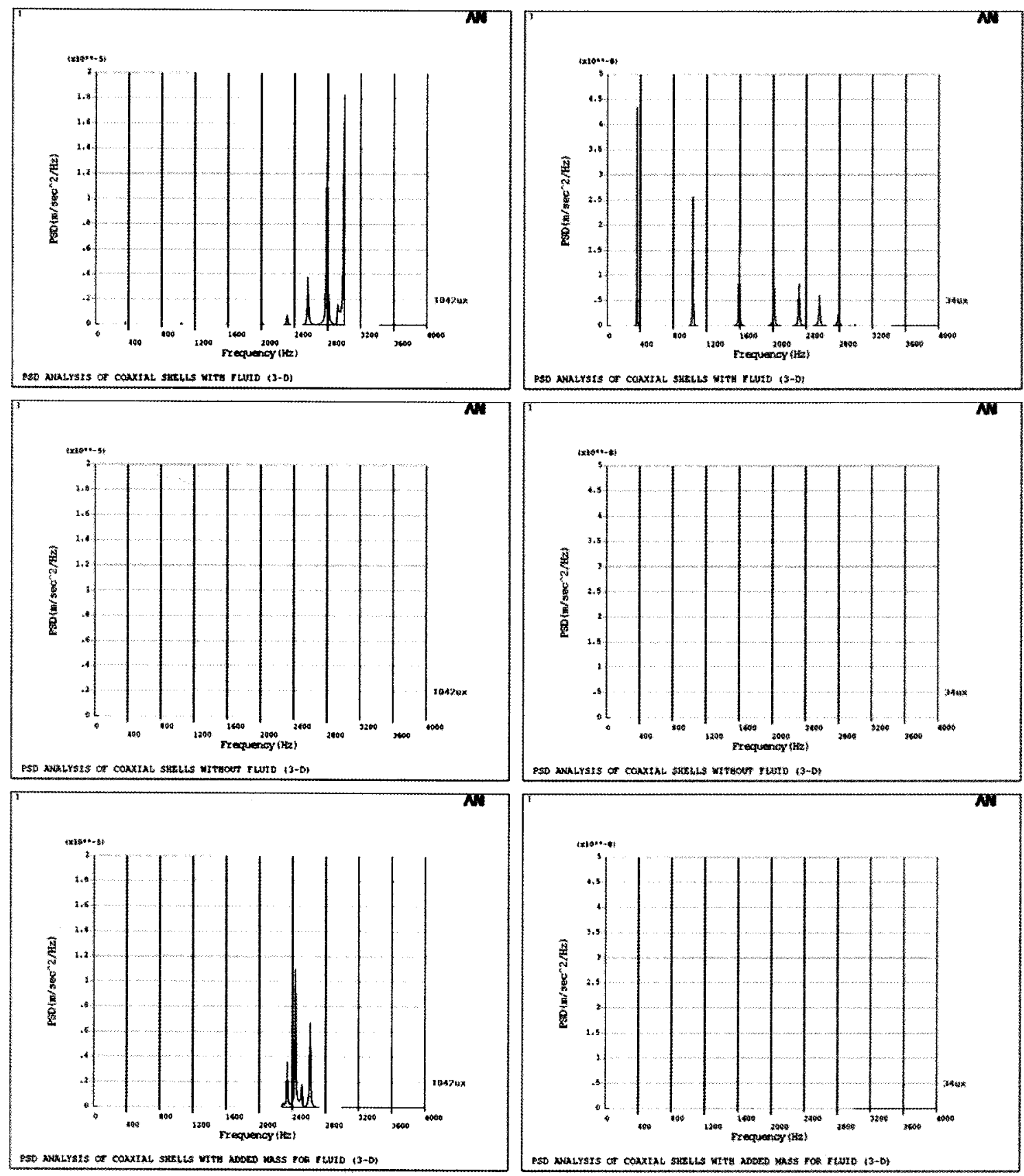

Fig. 16. Response PSDs from PSD Analysis

Table 2. Response Summaries from PSD Analysis

\begin{tabular}{l|c|c|c|c}
\hline \multirow{2}{*}{ Model } & \multicolumn{2}{|c|}{ Maximum deflection $(\mathrm{m})$} & \multicolumn{2}{c}{ Maximum equivalent stress (Pa) } \\
\cline { 2 - 5 } & Inner shell & Outer shell & Inner shell & Outer shell \\
\hline With fluid & $.210 \mathrm{E}-4$ & $.236 \mathrm{E}-4$ & $.907 \mathrm{E} 7$ & $.127 \mathrm{E} 8$ \\
\hline Without fluid & $.203 \mathrm{E}-4$ & $.203 \mathrm{E}-4$ & $.499 \mathrm{E} 7$ & $.495 \mathrm{E} 7$ \\
\hline $\begin{array}{l}\text { With added } \\
\text { mass for fluid }\end{array}$ & $.103 \mathrm{E}-3$ & $.103 \mathrm{E}-3$ & $.253 \mathrm{E} 8$ & $.251 \mathrm{E} 8$ \\
\hline
\end{tabular}

the responses are generally lowered.

The displacements at the node where the pulse is applied and at the node of the inner shell are shown in Figure 17, which shows that there is a small difference of responses between models. The maximum deflections from the transient analysis are shown in Table 3. As indicated in the table, the deflection is almost the same irrespective of the fluid model. If the fluid is not considered in the model, the response after the pulse is applied decreases very rapidly. But if the fluid is considered, the response due to 

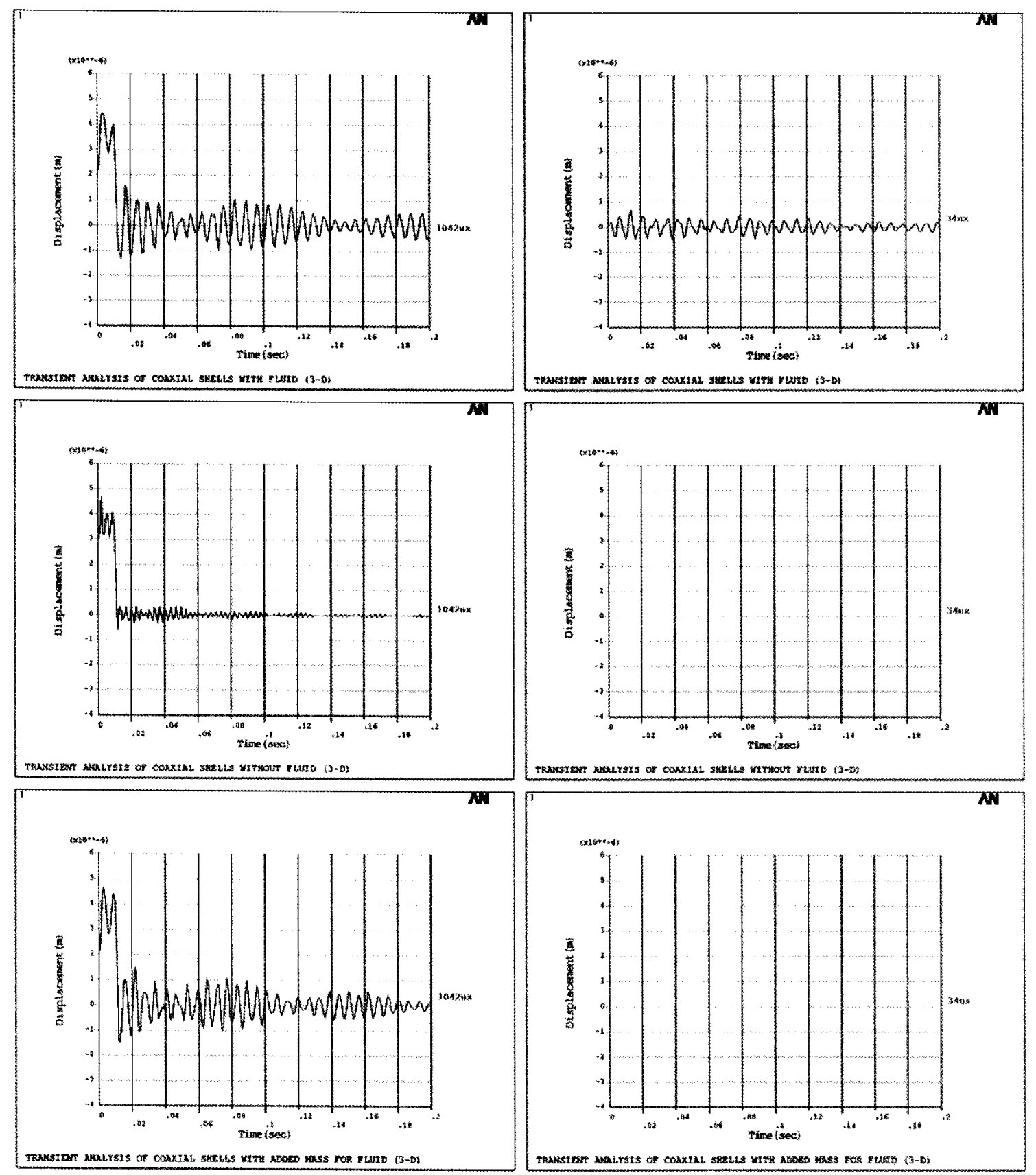

Fig. 17. Displacement Time Histories from Transient Analysis for 3-D Model

the pulse appears for a long time. As shown in Figure 17, there is no displacement at the node of the inner shell if the fluid is not included in the model because there is no load path from the outer shell where the pulse is applied to the inner shell via couplings between shell and fluid.

The same kind of transient analyses are performed for the 2-dimensional axisymmetric model. The displacements at corresponding nodes are shown in Figure 18. By comparing displacements between 3-dimensional and 2-dimensional models, it is not clear that the 2-dimensional axisymmetric model can simulate the transient analysis.

The displacements and velocities from the harmonic analysis at the node where the unit load is applied are shown in Figure 19, which shows that there is a difference of response between models due to the different modal characteristics. If the fluid is not considered in the model, the responses are generally lower than those for the model with fluid. But if the fluid is considered, the responses are almost the same irrespective of the fluid model representations such as added mass or fluid mass. 

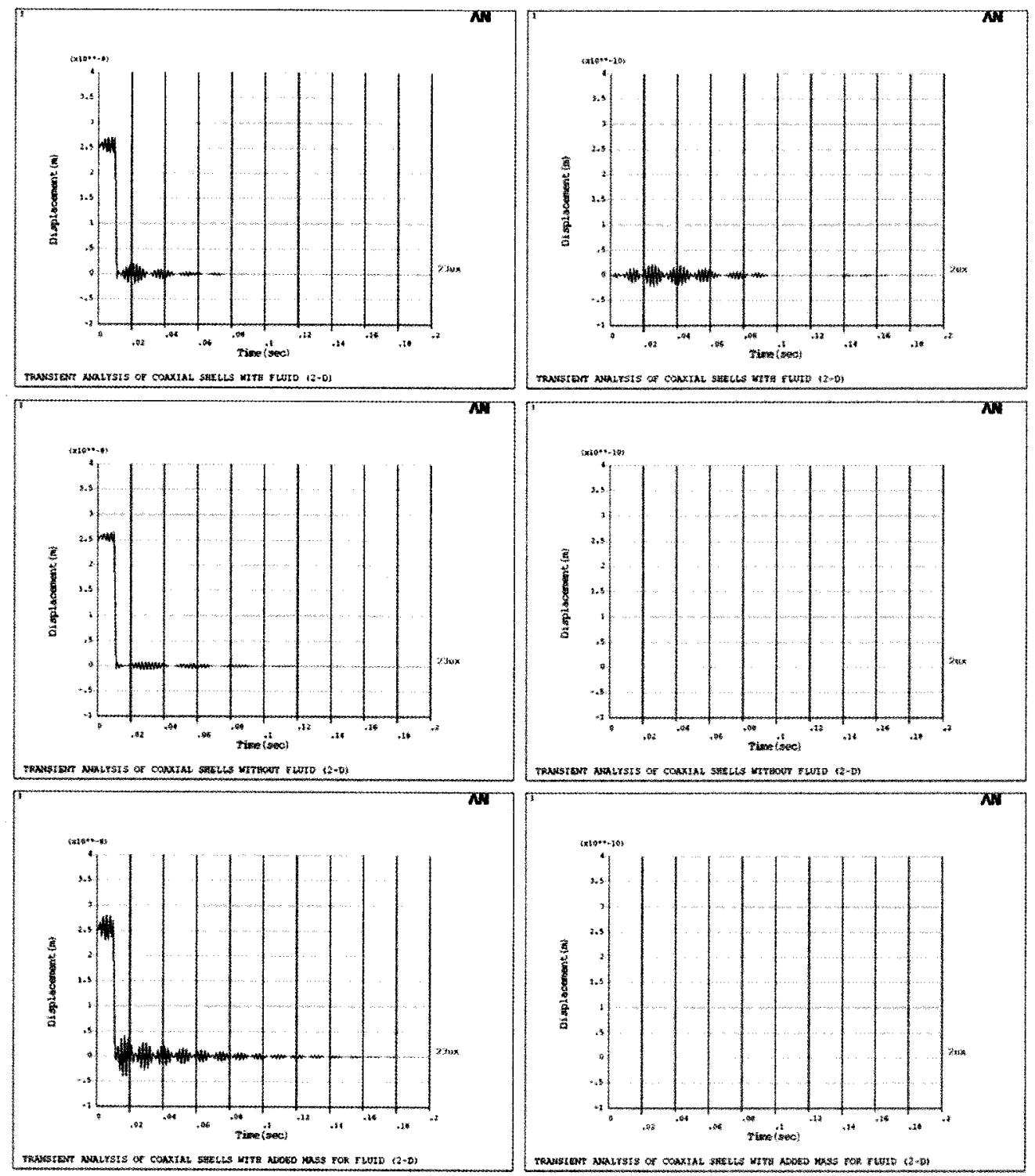

Fig. 18. Displacement Time Histories from Transient Analysis for 2-D Model

Table 3. Response Summaries from Transient Analysis

\begin{tabular}{|c|c|c|c|}
\hline \multirow{2}{*}{\multicolumn{2}{|c|}{ Model }} & \multicolumn{2}{|c|}{ Maximum responses } \\
\hline & & Displacement (m) & Time (sec) \\
\hline \multirow{3}{*}{ 3-D } & With fluid & $0.443844 \mathrm{E}-05$ & $0.40000 \mathrm{E}-02$ \\
\hline & Without fluid & $0.469215 \mathrm{E}-05$ & $0.20000 \mathrm{E}-02$ \\
\hline & $\begin{array}{l}\text { With added } \\
\text { mass for fluid }\end{array}$ & $0.465746 \mathrm{E}-05$ & $0.30000 \mathrm{E}-02$ \\
\hline \multirow{3}{*}{ 2-D } & With fluid & $0.272461 \mathrm{E}-07$ & $0.80000 \mathrm{E}-02$ \\
\hline & Without fluid & $0.265461 \mathrm{E}-07$ & $0.10000 \mathrm{E}-01$ \\
\hline & $\begin{array}{l}\text { With added } \\
\text { mass for fluid }\end{array}$ & $0.281476 \mathrm{E}-07$ & $0.60000 \mathrm{E}-02$ \\
\hline
\end{tabular}

\section{CONCLUSIONS}

Several representations of fluid were made for modal analysis of coaxial shells with fluid-filled annulus. Their modal characteristics are compared among several different models and they are used for generating dynamic responses due to response spectrum and power spectral density. Also, harmonic analysis and transient analysis with unit input load are performed for various models and the responses are compared, generating the following conclusions:

- The effect of fluid on the frequencies is more significant for out-of-phase mode and inner shell than in-phase mode and outer shell.

- Representing fluid by added mass gives higher frequencies

NUCLEAR ENGINEERING AND TECHNOLOGY, VOL.41 NO.10 DFCEMBER 2009 

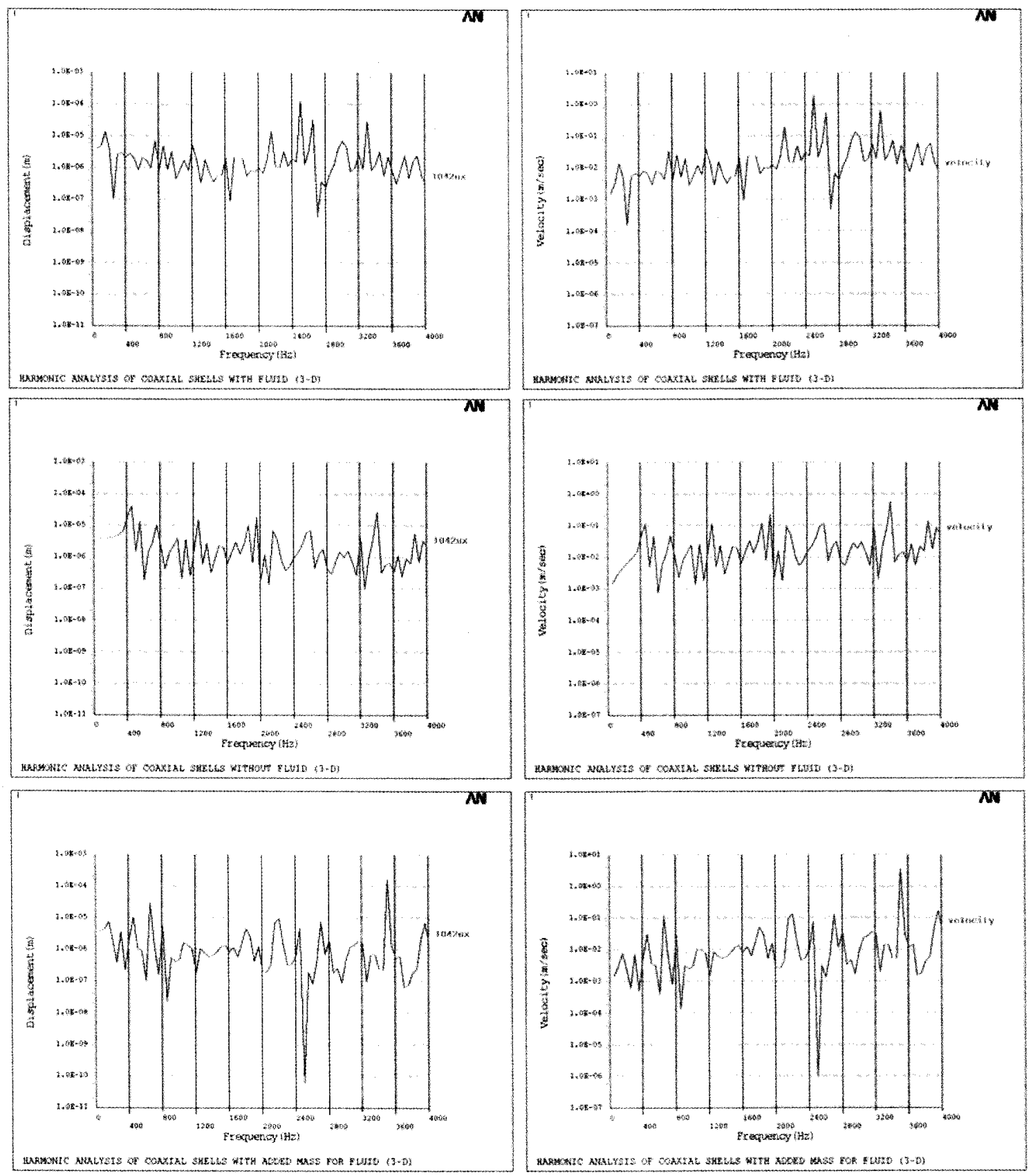

Fig. 19. Displacement and Velocity Time Histories from Harmonic Analysis

for in-phase modes and lower frequencies for out-ofphase modes.

- The axisymmetric-harmonic element is found to be a very efficient way to investigate the modal characteristics, suggesting the use of this element instead of a 3dimensional element for modal analysis.

- The axisymmetric model is not recommended for dynamic analysis except modal analysis.

- Failing to consider fluid coupling effect besides added mass for the response spectrum, PSD, transient and harmonic analyses may give unconservative results, which should not be neglected in the structural integrity assessment for the nuclear components.

\section{REFERENCES}

[ 1 ] Song, S.H., Jhung, M.J. "Experimental Modal Analysis on the Core Support Barrel of Reactor Internals Using a Scale Model," KSME International Journal, Vol.13, No.8, pp.585594 (1999).

[2] ANSYS, Inc., Theory Reference for ANSYS and ANSYS 
Workbench Release 11.0, Canonsburg, PA (2007).

[3] Jhung, M.J., Hwang, W.G., "Seismic Behavior of Fuel Assembly for Pressurized Water Reactor," Structural Engineering and Mechanics, Vol.2, No.2, pp.157-171 (1994).

[4] Blevins, R.D., Flow-Induced Vibration, $2^{\text {nd }}$ ed., Van Nostrand Reinhold, New York (1990).

[ 5 ] Jhung, M.J., Hwang, W.G., "Seismic Response of Reactor Vessel Internals for Korean Standard Nuclear Power Plant," Nuclear Engineering and Design, Vol.165, pp.5766 (1996).
[6] Caughey, T.K., "Classical Normal Modes in Damped Linear Dynamic Systems," Journal of Applied Mechanics, Vol.27, pp.269-271 (1960).

[ 7 ] Wilson, E.L., Pensien, J., "Evaluation of Orthogonal Damping Matrices," International Journal for Numerical Methods in Engineering, Vol.4, pp.5-10 (1972).

[8] Grimes, R.G., Lewis, J.G., and Simon, H.D., "A Shifted Block Lanczos Algorithm for Solving Sparse Symmetric Generalized Eigenproblems," SIAM Journal on Matrix Analysis and Applications, Vol.15, No.1, pp.228-272 (1994). 